\title{
812 ERYTHEMA NODOSUM-LIKE TOXICITY IN AN IMMUNOTHERAPY TREATED PATIENT IS ACCOMPANIED BY OLIGOCLONAL MEMORY ACTIVATED CD4 T CELLS
}

Xiaopeng Sun*, Margaret Axelrod, Yu Wang, Sanchez Violeta, Paula Gonzalez-Ericsson, Douglas Johnson, Justin Balko. Vanderbilt University Medical Center, Nashville, TN, United States

Background Immune checkpoint inhibitors (ICIs) are increasingly used to treat advanced malignancy but can be associated with immune related adverse events (irAE). Here we present a case report of a rare dermatologic toxicity occurring in a melanoma patient with isolated brain metastasis. After surgical resection, the patient was treated with ipilimumab (anti-CTLA4) and nivolumab (anti-PD-1) combination therapy followed by single agent nivolumab with ongoing, excellent response. During nivolumab, the patient developed an erythema nodosum (EN)-like irAE. The condition resolved after potassium iodine treatment and nivolumab therapy was resumed. To understand the pathogenesis of this irAE, we examined samples from this patient's blood, brain metastasis and tissue biopsy of the EN toxicity.

Methods RNA and T cell receptor (TCR) sequencing on the patient's brain metastasis and site of irAE were performed. We also performed RNA sequencing on 3 non-ICI EN patients. RNA in situ hybridization (RNAish) for CD4, CD8 and granzyme B, and the most abundant TCR identified was conducted on the patient's site of toxicity. Single cell RNA/TCR sequencing was carried out on the patient's peripheral blood mononuclear cells (PBMC) at baseline, 3 weeks after ipilimu$\mathrm{mab}$ and nivolumab combination therapy, during EN toxicity and after resolution.

Results RNAish showed that the most abundant TCR (20\% of total TCR sequencing reads at the site of toxicity) colocalized with CD4 at the site of toxicity. According to CIBERSORT deconvolution, the site of toxicity had high memory activated CD4 T cells and low M2 macrophage infiltration, which is different from the brain metastasis and non-ICI-induced EN cases. Compared to non-ICI EN, the EN skin biopsy was also enriched for interferon response and inflammation related genes. In the peripheral blood, cytotoxic CD8 T cells clonally expanded during EN toxicity, accompanied by a decrease in naïve/memory CD4 T cells. The TCR repertoire in the site of toxicity did not overlap with that in the tumor or PBMC.

Conclusions We found oligoclonal memory activated CD4 T cells are enriched at the site of toxicity, suggesting their association with EN toxicity. The unique TCR repertoire, gene expression profile and immune cell composition at the site of toxicity could indicate that the EN toxicity is distinct from the anti-tumor immunity and analogous non-ICI autoimmunity. Future work will focus on determining the antigen for this irAE and determining its relevancy to other skin toxicities and EN autoimmune conditions.

Ethics Approval IRB 100178 and 161485

Consent Approval under IRB 100178

http://dx.doi.org/10.1136/jitc-2021-SITC2021.812 